\title{
Members of the Myocyte Enhancer Factor 2 Transcription Factor Family Differentially Regulate $B d n f$ Transcription in Response to Neuronal Depolarization
}

\author{
Michelle R. Lyons, Charlotte M. Schwarz, and Anne E. West \\ Department of Neurobiology, Duke University Medical Center, Durham, North Carolina 27710
}

\begin{abstract}
Transcription of the gene encoding Brain-Derived Neurotropic Factor (BDNF) is induced in response to a wide variety of extracellular stimuli via the activation of a complex array of transcription factors. However, to what degree individual transcription factors confer specificity upon the regulation of $B d n f$ is poorly understood. Previous studies have shown that members of the myocyte enhancer factor 2 (MEF2) transcription factor family bind a regulatory element upstream of $B d n f$ promoter I and associate with an unknown binding site in $B d n f$ promoter IV. Here we identify calcium-response element 1 as the MEF2 binding site in promoter IV of the $B d n f$ gene and determine the requirements for individual MEF2 family members in Bdnf regulation. MEF2A, MEF2C, and MEF2D are all highly expressed in embryonic rat cortical neurons; however, only the Mef2c gene encodes an MEF2 splice variant that lacks the $\gamma$ repressordomain. We find that MEF2C variants lacking the $\gamma$-domain are particularly sensitive to activation by membrane depolarization, raising the possibility that the MEF2s may differentially contribute to activity-regulated gene expression. We find that only knockdown of MEF2C significantly impairs membrane depolarization-induced expression of $B d n f$ exon IV. By contrast, knockdown of MEF2D significantly enhanced depolarization-induced expression of $B d n f$ exon I. Together, these data show that individual members of the MEF2 family of transcription factors differentially regulate the expression of $B d n f$, revealing a new mechanism that may confer specificity on the induction of this biologically important gene.
\end{abstract}

\section{Introduction}

Neuronal activity-regulated gene transcription plays an important role in converting sensory information into long-lasting changes in brain function (Lyons and West, 2011). The gene encoding the secreted neurotrophin brain-derived neurotrophic factor (BDNF) is a key player in this process, as it performs essential roles in modulating synaptic development and function (Loebrich and Nedivi, 2009). The Bdnf gene contains nine alternative promoters that are thought to facilitate cell type-, region-, and stimulus-specific regulation of $B d n f$ transcription (Timmusk et al., 1993; Loebrich and Nedivi, 2009). Promoters I and IV are both highly responsive to neuronal activity; however, transcripts initiated from promoter IV (pIV) are most abundant in developing neurons (Timmusk et al., 1993; Lyons and West, 2011).

Studies examining transcription factor binding sites within $\mathrm{pI}$ and $\mathrm{pIV}$ of the $B d n f$ gene have identified multiple calcium re-

Received Feb. 3, 2012; revised June 28, 2012; accepted July 25, 2012.

Author contributions: M.R.L. and A.E.W. designed research; M.R.L., C.M.S., and A.E.W. performed research; M.R.L. and C.M.S. analyzed data; M.R.L. wrote the paper.

This work was supported by the Ruth K. Broad Biomedical Research Foundation (M.R.L.), and by grants to A.E.W. from the Whitehall Foundation and the NIH (Grant R01DA022202). We thank Dr. Tod Gulick for the Gal4-MEF2C plasmids, Dr. Michael E. Greenberg for the Bdnf promoter I reporter plasmids, and Lauren Burch for technical assistance.

The authors declare no competing financial interests.

Correspondence should be addressed to Anne E. West, Department of Neurobiology, DUMC Box 3209, Bryan Research Building, 301D, Durham, NC 27710. E-mail: west@neuro.duke.edu.

DOI:10.1523/JNEUROSCI.0534-12.2012

Copyright $\odot 2012$ the authors $\quad 0270-6474 / 12 / 3212780-06 \$ 15.00 / 0$ sponse elements (CaREs) that are required for the L-type voltagegated calcium channel (L-VGCC)-dependent induction of $B d n f$ expression driven by membrane depolarization (Lyons and West, 2011; Pruunsild et al., 2011). Calcium response factor (CaRF) is a binding protein for the CaRE1 element within Bdnf pIV (Tao et al., 2002), and levels of $B d n f$ exon IV mRNA are significantly reduced in the cerebral cortex of CaRF knock-out mice (McDowell et al., 2010). However, cultured CaRF knock-out neurons show normal upregulation of $B d n f$ exon IV in response to membrane depolarization. Thus, these data raised the possibility that additional CaRE1 binding proteins may mediate the activity of this element following membrane depolarization.

In this study, we identify the myocyte enhancer factor 2 (MEF2) family of transcription factors as mediators of membrane depolarization-inducible, CaRE1-dependent transcription from $B d n f$ pIV. The MEF2 family is comprised of four separate gene products (MEF2A-D) that are differentially expressed spatially and temporally in the brain (Lyons et al., 1995). MEF2A, MEF2C, and MEF2D are coexpressed in embryonic cortical neurons, and the $\mathrm{Mef} 2 \mathrm{c}$ gene encodes multiple MEF2C splice variants that we show are differentially responsive to membrane depolarization. We demonstrate that knockdown of MEF2C selectively reduces membrane depolarization-induced expression of $B d n f$ exon IV, whereas induction of $B d n f$ exon I expression is potentiated by knockdown of MEF2D. Our data suggest a novel mechanism of specificity in the transcriptional regulation of $B d n f \mathrm{pIV}$ via the actions of distinct transcription factors within a single family. We propose that this transcriptional diversity may enable 
A

plV-luc:

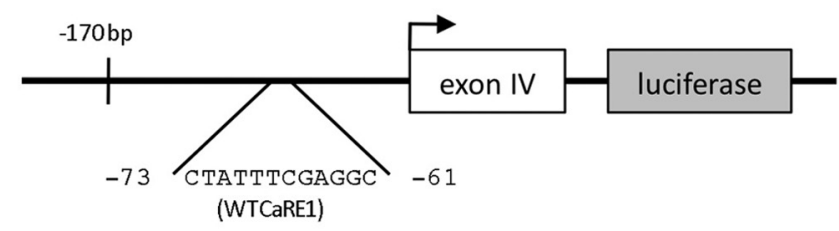

pIV(mCaRE1)-luc:
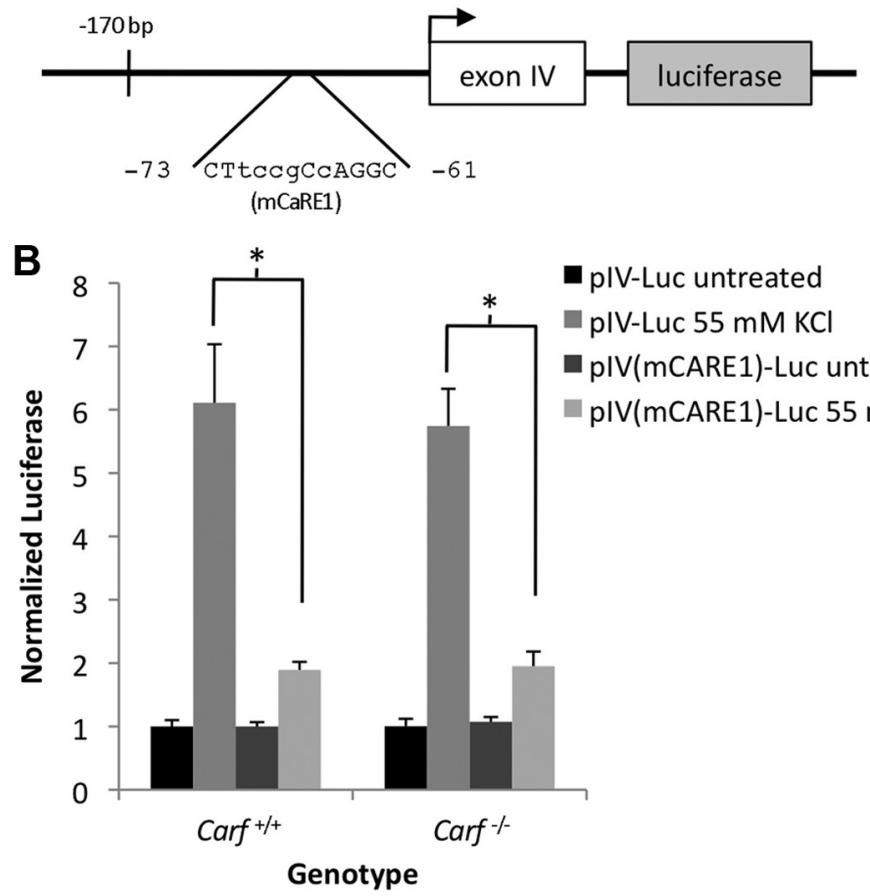

Figure 1. CaRE1 is required for membrane depolarization-induced activation of $B d n f$ promoter IV in CaRF knock-out neurons. $\boldsymbol{A}$, Schematic of the $B d n f$ plV-luciferase reporter plasmids used. The numbers indicate the position of the promoter $5^{\prime}$ end relative to the initiation site of transcription. pIV-Luc (Tao et al., 2002) contains the proximal $170 \mathrm{bp}$ of Bdnf plV; plV(mCaRE1)-Luc has the CaRE1 site mutated to a sequence that does not bind CaRF. $\boldsymbol{B}$, Mutation of the CaRE1 element leads to a reduction in membrane depolarization-induced Bdnf pIV activity in both $\mathrm{Carf}^{+/+}$and $\mathrm{Carf}^{-/-}$cortical neurons. Single pup cultures were independently transfected and treated with $\mathrm{KCl} . n=2 \mathrm{~K} 0$ and 3 WT pups. ${ }^{*} p<0.05$, pIV(m(aRE1) compared with pIV.

(Charles River Laboratories), or E18/19 CD1 rat embryos (Charles River), as described previously (Tao et al., 2002; McDowell et al., 2010). For luciferase assays, neurons or HEK $293 \mathrm{~T}$ cells (ATCC) were transfected with calcium phosphate (Tao et al., 2002). Cotransfection of TK-renilla luciferase (Promega) was used to control for transfection efficiency and sample handling. For neurons, lysates were harvested on day in vitro 5-6 (DIV5-6) after overnight treatment with $1 \mu \mathrm{M}$ sodium channel inhibitor TTX (Sigma) \pm 6 h exposure to $55 \mathrm{~mm}$ extracellular $\mathrm{KCl}$, which activates L-VGCCdependent transcription of $B d n f$ (Tao et al., 2002).

Electrophoretic mobility shift assay. In vitro transcribed/translated MEF2A and MEF2D were incubated with a radiolabeled double-stranded MEF response element (CTCTAAAAATAACCCA) for electrophoretic mobility shift assay (EMSA) as described previously (Pfenning et al., 2010). Unlabeled double-stranded competition probes (CaRE1: GTGTCTATTTCGAGGCAGAGGA; cCaRE: GTGTYCARAACGAGGCAGAGG) were added in 10-, 100-, or 1000-fold molar excess. Gels were dried and visualized by PhosphorImager, and shift intensities were quantified relative to probe intensity in the same lane using ImageQuant (GE Healthcare).

RNA interference plasmids and quantitative $R T-P C R$. An shRNAmir plasmid targeting rat MEF2C in the doxycycline-inducible lentiviral vector pTRIPZ (V2THS_151686, Thermo Scientific) was used in luciferase assays. Doxycycline $3 \mu \mathrm{M}$ was added for $72 \mathrm{~h}$ after transfection to induce shRNA expression. MEF2 knock-down constructs targeting rat MEF2A, MEF2C, or MEF2D in the vector pLKO.1 (TRCN0000095959, TRCN0000012068, and TRCN0000085268) were obtained (Thermo Scientific) and transfected for luciferase assays or packaged as lentiviral particles in $293 \mathrm{~T}$ cells. Cultured rat cortical neurons were infected on DIV1. RNA was harvested on DIV7 for cDNA synthesis and quantitative SYBR green PCR (ABI). Intron spanning primers were used for all genes tested. Gapdh mRNA levels

neurons to finely tune their cellular responses to environmental stimuli.

\section{Materials and Methods}

Plasmids. Wild-type or CaRE1 mutant Bdnf pIV firefly luciferase reporter plasmids were previously called p3-170Luc and p3-170Luc3-4, respectively (Tao et al., 2002). The following plasmids were previously described: cCaRE, mCaRE, CaRE1 (McDowell et al., 2010), MEF2 response element (MRE) (Flavell et al., 2006), Bdnf pI-Luc (-6143), and pI-Luc (-4495) (Flavell et al., 2008) luciferase reporter plasmids; Gal4 fusions with human MEF2C $\alpha_{1}$, MEF2C $\alpha_{1} \beta, \operatorname{MEF} 2 C \alpha_{1} \gamma$, MEF2C $\alpha_{1} \beta \gamma$, or MEF2C $\alpha_{1} \beta \Delta \gamma$ (Zhu and Gulick, 2004); the UAS-luciferase plasmid (Tao et al., 2002); mammalian expression vectors for CaRF (Tao et al., 2002), MEF2A and MEF2D (Flavell et al., 2006), and CREB (Kornhauser et al., 2002); and the shRNA plasmids targeting mouse MEF2A and MEF2D used in neuronal transfection experiments (Flavell et al., 2006). The $B d n f \mathrm{pIV}(\Delta \mathrm{CaRE} 1-2 \mathrm{xUAS})$-Luc reporter was generated using around the world PCR with primers that replace CaRE1 (GTGTCTATTTGCAGGCAG) with two tandem copies of the UAS (CGGAAGACTCTCCTCCG).

Cell culture, transfection, and luciferase assays. Neuron-enriched cultures were generated from cortices of male and female P0 Carf ${ }^{+/+}$or Carf $^{-1-}$ littermates (McDowell et al., 2010), E16 CD1 mouse embryos were used as a normalizing control. The PCR primers used were as follows: Gapdh: forward, CATGGCCTTCCGTGTTCCT; reverse, TG ATGTCATCATACTTGGCAGGTT; $M e f 2 a$ : forward, TCAAGCCACA CAACCTCTTG; reverse, CAGCATTCCAGGGGAAGTAA; $M e f 2 b$ : forward, AGAGGGTCCTGGAGAGAAGC; reverse, AGGTGGCTCG GAGAGAAGAT; Mef2c: forward, CCAAATCTCCTCCCCCTATG; $M e f 2 c \gamma$ : forward, TCAAATCTCTCCCTGCCTTC; reverse, CTCCCA TCGTAGGAACTGCT; $M e f 2 d$ : forward,CAGCAGCCAGCACTACAG AG; reverse, GGCAGGGATGACCTTGTTTA; Fos: forward, TTTATC CCCACGGTGACAGC; reverse, CTGCTCTACTTTGCCCСTTCT; $B d n f$ exon IV: forward, CGCCATGCAATTTCCACTATCAATAA; reverse, GCCTTCATGCAACCGAAGTATG; and $B d n f$ exon I: forward, AGTCTC CAGGACAGCAAAGC; reverse, GCCTTCATGCAACCGAAGTA.

Statistics. All data presented are the average of at least two independent measurements. Data were analyzed by a Student's unpaired $t$ test, and $p<0.05$ was considered significant. Bar graphs show mean values, and all error bars show SEM.

\section{Results}

Membrane depolarization drives CaRE1-dependent transcription of $B d n f$ exon IV (Tao et al., 2002). Although CaRF binds to CaRE1 (Tao et al., 2002; Pfenning et al., 2010) and CaRF knock-out mice have 
reduced cortical Bdnf exon IV mRNA levels, loss of CaRF does not impair membrane depolarization-induced $B d n f$ exon IV transcription (McDowell et al., 2010). To test whether CaRE1 is required for membrane depolarization-induced transcription of $B d n f$ exon IV in the absence of CaRF, we transfected cultured $\mathrm{Carf}^{+/+}$or $\mathrm{Carf}^{-/-}$ cortical neurons with $B d n f$ pIV reporter plasmids (Fig. 1A). In both $\mathrm{Carf}^{+/+}$and Carf $^{-1-}$ neurons, membrane depolarization robustly induced transcription from the wild-type pIV plasmid (Fig. 1B). Mutation of the CaRE1 binding site significantly inhibited membrane depolarization-induced luciferase expression in both $\mathrm{Carf}^{+/+}$and $\mathrm{Carf}^{-/-}$neurons, demonstrating CaRE1 dependence. These data strongly suggest that CaRE1-binding proteins other than CaRF mediate the activity of this element in response to membrane depolarization.

Alignment of the consensus high-affinity CaRF binding site (cCaRE) (Pfenning et al., 2010) with the Bdnf CaRE1 sequence revealed that the cCaRE closely matches only the $3^{\prime}$ half of CaRE1 (Fig. 2A). By contrast, the $5^{\prime}$ side of CaRE1 aligns well with the consensus binding motif for the MEF2 family of transcription factors (Gossett et al., 1989). MEF2A-D are highly expressed in the nervous system, where they play important roles in neuronal activity-dependent transcription and synaptic development (Shalizi and Bonni, 2005). MEF2 has been shown by chromatin immunoprecipitation to associate with $B d n f$ pIV in vivo; however, the site of binding was not identified (Hong et al., 2008). Thus, we considered the possibility that MEF2 may bind CaRE1. Recombinant MEF2A/MEF2D heterodimers were tested by competition EMSA for their ability to bind CaRE1. Addition of oligonucleotides containing the CaRE1 site competed binding of MEF2 to a consensus MRE (Gossett et al., 1989), indicating that MEF2 can bind CaRE1 (Fig. 2 B, C). Quantification of the competition revealed that a 100 -fold excess of unlabeled MRE competed $47.7 \pm 5 \%$ of the binding to the radiolabeled MRE probe, whereas it took a 1000-fold excess of unlabeled CaRE1 to reach a similar level of competition $(48.4 \pm 4.3 \%)$. Thus, MEF2A/ MEF2D has an $\sim 10$-fold higher affinity for the MRE relative to CaRE1. We found that oligonucleotides containing the consensus CaRF binding site, which has no homology to the MEF2 binding site, were unable to compete for MEF2 binding.

We next asked whether MEF2 can drive CaRE1-dependent $B d n f$ transcription. Overexpression of either MEF2A/D or CaRF in HEK 293T cells was sufficient to drive expression of luciferase from a wild-type $B d n f$ pIV reporter plasmid. By contrast, neither MEF2 nor CaRF activated a $B d n f$ pIV reporter in which the CaRE1 site was mutated, demonstrating the CaRE1 dependence of the activity of both factors at this promoter (Fig. 2C). Transcription from both the wild-type and CaRE1 mutant plasmids was promoted equally by overexpressed CREB, which binds $B d n f$ pIV at a site (CaRE3/CRE) distinct from CaRE1 (Tao et al., 1998).

To determine whether endogenous MEF2 contributes to CaRE1dependent transcription in neurons, we assessed the effect of RNA interference (RNAi)-mediated knockdown of neuronal MEF2 on

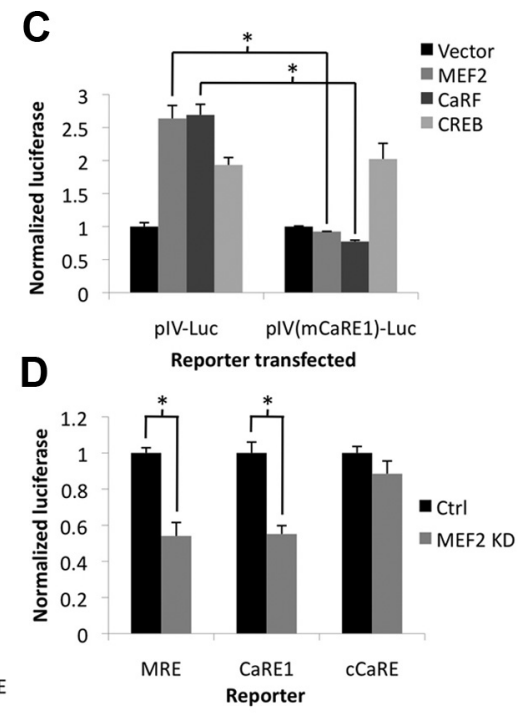

Figure 2. MEF2 binds to CaRE1 and drives CaRE1-dependent gene transcription. $\boldsymbol{A}$, Alignment of the consensus CaRF binding (T) Bdnf pIV CaRE1 element (Ta0 et al., 2002), and a canonical MRE (Gossett et al., 1989). Vertical lines 列 作 作

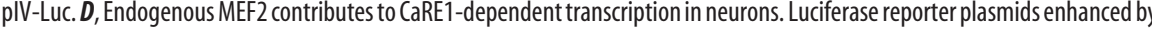
MRE, CaRE1, or cCaRE were transfected into cultured mouse cortical neurons along with shRNA vectors that knock down (KD) 政

luciferase expression from cotransfected MRE, CaRE1, or cCaRE reporter plasmids. Neurons were transfected with either a control shRNA plasmid, or shRNA-expressing plasmids that knockdown expression of MEF2A and MEF2D (Flavell et al., 2006). Compared with control neurons, knockdown of MEF2 significantly reduced luciferase expression from a reporter enhanced by three copies of the MRE, demonstrating the efficacy of the RNAi (Fig. 2D). Knockdown of MEF2A/MEF2D also significantly reduced luciferase expression from the reporter enhanced by three copies of the wild-type CaRE1 site. By contrast, MEF2 knockdown had no effect on luciferase expression from a reporter enhanced by the consensus CaRF binding site (Fig. 2D). These data implicate MEF2 along with CaRF as a CaRE1-dependent transcriptional regulator in neurons.

Knockdown of MEF2A and MEF2D in cultured hippocampal neurons has previously been shown to reduce L-VGCC-dependent induction of $B d n f$ (Flavell et al., 2008). However, whereas Mef $2 a$ and $M e f 2 d$ are the primary MEF2 family members expressed in the hippocampus, Mef $2 c$ transcripts are the most highly expressed of the MEF2s in cortical neurons (Fig. $3 A, B$ ). Mef2 $b$ expression is negligible by comparison. MEF2A, MEF2C, and MEF2D all undergo alternative splicing of two domains called $\alpha$ and $\beta$; however, only MEF2C shows alternative splicing of the $\gamma$-domain. This is important because the $\gamma$-domain is a major site of regulation of the activity of the MEF2 transcription factors by neuronal signaling cascades (Shalizi et al., 2006). In cultured rat cortical neurons, only $48.4 \pm 4.3 \%$ of Mef $2 c$ transcripts contain the $\gamma$-domain, as determined by RT-PCR. To determine how differential splicing affects the membrane depolarization-dependent regulation of MEF2C activity, we overexpressed a series of Gal4-MEF2C splice-variant fusion constructs in cultured cortical neurons along with a UAS-luciferase reporter plas- 
A

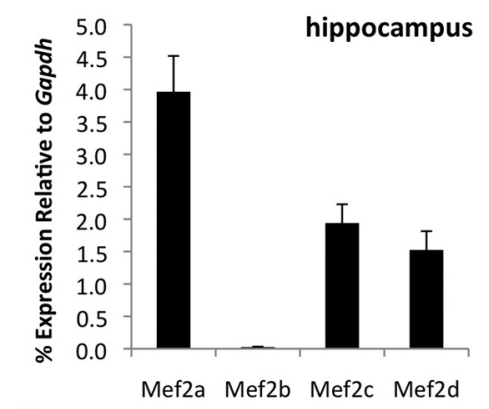

C

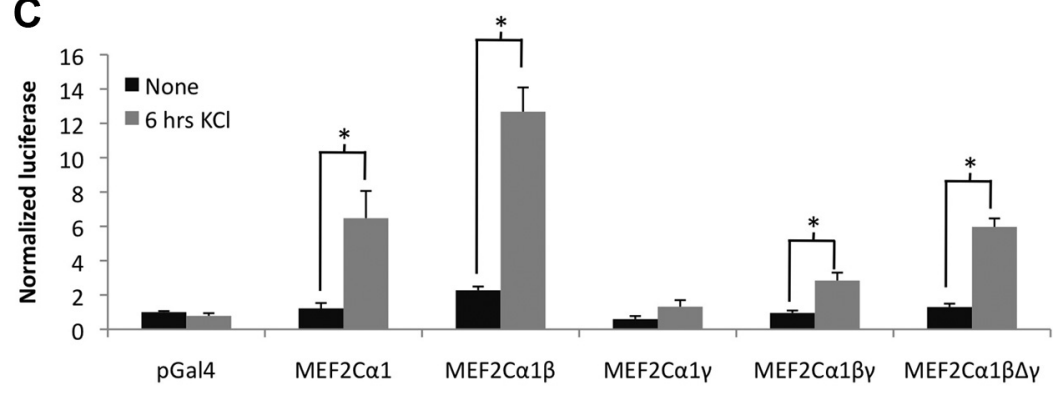

Reporter

B

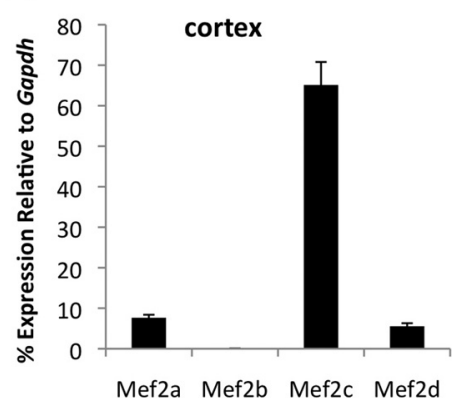

Figure 3. Region-specific expression and calcium-dependent regulation of MEF2s. $A, B$, Relative expression of MEF2 family members was determined by quantitative PCR from cultured embryonic rat hippocampal $(\boldsymbol{A})$ or cortical $(\boldsymbol{B})$ neurons. $n=4$. $\boldsymbol{C}$, $M E F 2 C$ splice variants are differentially responsive to membrane depolarization. Cultured embryonic rat cortical neurons were cotransfected with a pUAS-Luc reporter vector along with plasmids expressing Gal4 fusions of the indicated MEF2C splice variants. $\Delta \gamma, \mathrm{A} \gamma$-containing MEF2C variant with an S396A point mutation. The bars show the mean values from four independent experiments scaled to the untreated empty vector control from each day. In all graphs, error bars represent SEM. ${ }^{*} p<0.056 \mathrm{~h} \mathrm{KCl}$ versus none.

mid. Whereas the presence of the $\beta$-domain led to higher levels of membrane depolarization-induced transcription, the presence of the $\gamma$-domain significantly reduced activation of MEF2C (Fig. $3 C$ ). Consistent with evidence that phosphorylation of MEF2C at serine 396 within the $\gamma$-domain is a mechanism of the repressive activity of this domain (Zhu and Gulick, 2004; Shalizi et al., 2006), mutation of this residue to alanine increased membrane depolarization-induced transcription (Fig. 3C). These data suggest that MEF2C splice variants lacking the $\gamma$-domain, which are highly expressed in the developing cortex, are likely to be the most sensitive of the MEF2 transcription factors to membrane depolarization.

To determine whether individual members of the MEF2 family differentially regulate $B d n f$ transcription, we used lentiviruses carrying shRNAs to selectively knock down MEF2A, MEF2C, or MEF2D (Fig. 4A). Knocking down MEF2 family members does not impair activity-regulated gene transcription in general because membrane depolarization-induced Fos expression was unaffected by any of the knockdowns (Fig. 4B). We found that knockdown of MEF2C, but not MEF2A or MEF2D, significantly reduced the membrane depolarization-dependent induction of $B d n f$ exon IV-containing transcripts (Fig. 4C). Either of two independent shRNAs targeting MEF2C impaired membrane depolarization-induced transcription from the $B d n f \mathrm{pIV}$-Luc reporter plasmid in transfected neurons, localizing the requirement for MEF2C to the proximal region of $B d n f$ pIV (Fig. 4D). To determine whether recruitment of MEF2C is sufficient to confer membrane depolarization-dependent regulation upon $B d n f \mathrm{pIV}$, we replaced the CaRE1 sequence in the $B d n f$ pIV-Luc reporter plasmid with two copies of the Gal4-UAS binding sequence $[B d n f$ pIV( $\Delta$ CaRE1-2xUAS)-Luc], which eliminated $\mathrm{KCl}$-dependent luciferase expression (Fig. $4 E$ ). However, coexpression of a Gal4-MEF2C $\alpha 1 \beta$ fusion protein, which is recruited to the altered binding site, rescued membrane depolarization-induced transcription. These data identify MEF2C as an important contributor to activitydependent induction of $B d n f$ pIV in developing cortical neurons.

Although exon IV-containing forms of $B d n f$ are the most abundant $B d n f$ transcripts in the developing cortex, $B d n f \mathrm{pI}$ is also highly activity responsive in neurons and MEF2D has been found bound to an element $4.8 \mathrm{~kb}$ upstream of $\mathrm{pI}$ that can regulate pI activity (Flavell et al., 2008). Unlike the effects on pIV, knockdown of MEF2C had no impact on membrane depolarizationinduced $B d n f$ exon I expression, and knockdown of MEF2A resulted in a small but statistically significant impairment of $B d n f$ exon I induction (Fig. 4F). By contrast, knockdown of MEF2D significantly enhanced Bdnf exon I induction (Fig. $4 F$ ). MEF2D knockdown also increased transcription from a luciferase reporter plasmid containing $B d n f$ promoter I, localizing the repressive effects of MEF2D to this genomic fragment (Fig. $4 G$ ). However, this enhancement did not require the upstream MEF2 binding element (Fig. 4G), indicating that the repressive effects of MEF2D are mediated by other gene regulatory elements within the proximal or distal region of $B d n f$ pI. Collectively, these data suggest that specific MEF2 family members have distinct effects on the regulation of different Bdnf gene promoters in response to membrane depolarization.

\section{Discussion}

MEF2 family members are highly expressed in the nervous system where they regulate calcium-dependent transcriptional programs that are important for synapse development (Flavell et al., 2006; Shalizi et al., 2006). $B d n f$ is among the transcriptional targets of MEF2 in hippocampal neurons (Flavell et al., 2008), and, by chromatin immunoprecipitation, endogenous MEF2 proteins have been found to associate both with an element in pIV and with a putative long-distance $B d n f$ enhancer element $4.8 \mathrm{~kb}$ upstream of pI (Flavell et al., 2008; Hong et al., 2008). However, the functional importance of the interactions of specific MEF2 family members with each of these binding sites was not known.

We find that two different CaRE1-associated proteins, CaRF and MEF2C, are each required for aspects of $B d n f$ pIV activity in cortical neurons. Multiplex regulation is an important principle of $B d n f$ transcription. Not only are there as many as nine alternative $B d n f$ promoters, $\mathrm{pIV}$ alone is regulated by at least eight families of transcription factors (Lyons and West, 2011; Pruunsild et al., 2011). Whereas in vivo studies that have deleted any single transcription factor have shown only a moderate impact on Bdnf transcription, deletion of just one of the calcium response elements in $B d n f$ pIV (the CaRE3/CRE site bound by CREB family members) significantly impairs activity-dependent $B d n f$ gene induction (Hong et al., 2008). Thus, diversity of transcription factor regulation may confer distinct modes of activation on the $B d n f$ gene and/or ensure a high safety factor for $B d n f$ induction following increases in intracellular calcium. Both CaRF and 
MEF2C share a similar expression profile with highest levels during early postnatal cortical development (Lyons et al., 1995; McDowell et al., 2010). The close proximity of their binding sites at CaRE1 raises the possibility that these two transcription factors could cooperate or compete for binding to $\mathrm{pIV}$, although this remains to be experimentally addressed in vivo. Future studies comparing the actions and regulation of $\mathrm{CaRF}$ and $\mathrm{MEF} 2 \mathrm{C}$ at $B d n f$ pIV may help to explain how differential expression, recruitment, or activation of specific transcription factors at single $B d n f$ regulatory elements may provide a mechanism of specificity for the regulation of this important gene.

The MEF2 genes show variant expression patterns across different cell types and tissues, which is likely to contribute to their differential biological functions (Lyons et al., 1995). However, in some places, such as neurons of the cerebral cortex, multiple MEF2 family members as well as multiple splice variants from the same Mef2 gene are coexpressed in the same neurons (Lyons et al., 1995). By using RNAi to selectively knock down expression of individual MEF2 family members, we have now shown in developing cerebral cortical neurons, which coexpress MEF2A, MEF2C, and MEF2D, that only $\mathrm{MEF} 2 \mathrm{C}$ is required for membrane depolarization-dependent transcriptional activation of $B d n f$ pIV. This selective requirement is not likely to be due to differential binding of MEF2C versus MEF2A and MEF2D to pIV, both because the MEF2s share a highly conserved DNA binding domain and because in vivo chromatin immunoprecipitation experiments have demonstrated the association of endogenous MEF2D with $B d n f$ pIV in cortex (Hong et al., 2008). Furthermore, the relative importance of MEF2C may be selective to cortical neurons because dual knockdown of MEF2A and MEF2D in hippocampal neurons was shown in a previous study to impair membrane depolarization-induced expression of $B d n f$ (Flavell et al., 2008). These data raise the possibility that the functional contributions of the MEF2s to the regulation of $B d n f$ may be context or cell type specific. We suggest that one way that selective functions of the MEF2s may arise is as a consequence of their differential sensitivity to activation by membrane depolarization. Only the Mef $2 c$ gene encodes variants that lack the $\gamma$-domain (Zhu and Gulick, 2004; Shalizi and Bonni, 2008), and we find that $\gamma$-lacking MEF2C variants are the most highly activated of the MEF2Cs upon membrane depolarization. In the developing cortex, Mef2c is by far the predominant Mef2 gene expressed (Fig. $3 B$ ), and approximately half of the Mef2c transcripts lack the $\gamma$-domain. Thus, the heightened sensitivity of $\gamma$-lacking MEF2C vari-
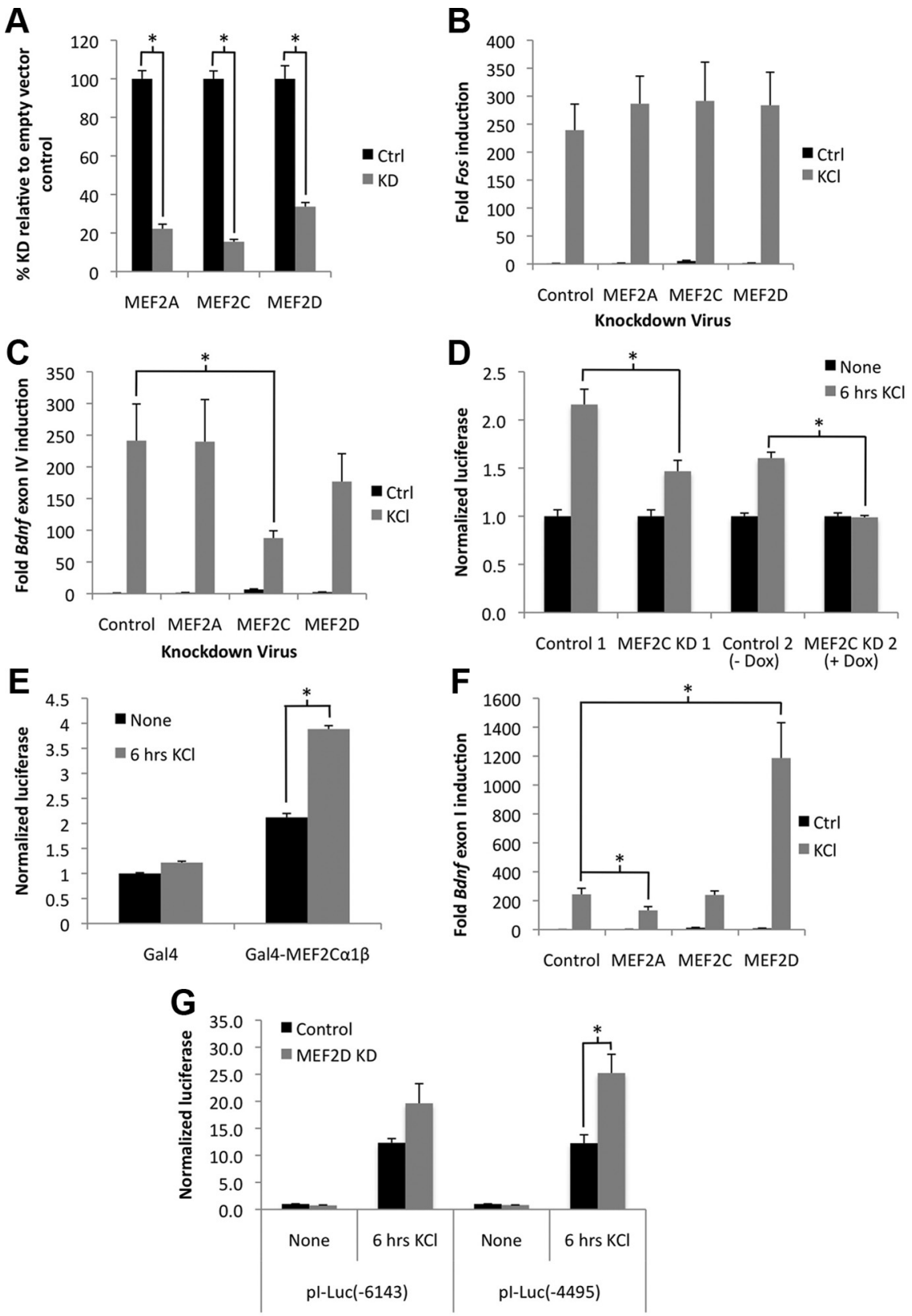

Figure 4. Differential regulation of endogenous Bdnf transcription by MEF2 family members in cortical neurons. $A$, Cultured embryonic rat cortical neurons were infected with lentiviruses containing shRNAs targeting individual MEF2 family members. Knockdown (KD) of endogenous mRNA levels was validated by quantitative PCR. MEF2 expression is normalized to levels in cells infected with the empty vector control viruses. $n=4 . B, C$, Cortical neurons were infected with the indicated lentiviruses then left untreated or stimulated with $55 \mathrm{~mm} \mathrm{KCl}$ for $3 \mathrm{~h}$. Endogenous Fos $(\boldsymbol{B})$ or Bdnf exon IV (C) mRNA levels were determined by quantitative PCR. D, Luciferase expression from Bdnf pIV-Luc cotransfected with the indicated plasmids. Control 1, Transfected with empty pLK0.1 vector; Control 2, transfected with pTRIPZ dox-inducible MEF2C shRNAmir in the absence of doxycycline. $\boldsymbol{E}$, Luciferase expression from the $B d n f p I V(\Delta$ CaRE1-2xUAS)-Luc plasmid cotransfected with the indicated Gal4 expression plasmids. F, Quantitative PCR for Bdnf exon I in cortical neurons infected with the indicated lentiviruses. G, Luciferase expression from a Bdnf pl-Luc reporter plasmid ( -6143$)$ or a truncated reporter lacking the $-4.8 \mathrm{~kb}$ upstream MEF2 binding site $(-4495)$ contransfected with the indicated plasmids. $n=3-4 .{ }^{*} p<0.05$ versus Control (Ctrl).

ants to L-VGCC activation may be particularly important for coupling the induction of activity-regulated genes like $B d n f$ to environmental stimuli during cortical development.

Interestingly, although knockdown of MEF2D in cortical neurons had no effect on $B d n f$ pIV regulation, membrane depolarization-dependent induction of $B d n f$ exon I-containing transcripts was strongly upregulated in neurons that lack this, but 
not in other MEF2 family members. Because the MEF2s are known to bind corepressors as well as coactivators (Shalizi and Bonni, 2008), it is possible that selective interactions between MEF2D and a transcriptional corepressor could be important for dampening the transcriptional activity of $B d n f \mathrm{pI}$ in developing cortical neurons. This would be particularly interesting because unlike $B d n f$ pIV, which is highly active and inducible in young neurons, $B d n f \mathrm{pI}$ is relatively inactive until adulthood, when its inducibility is markedly elevated via unknown mechanisms (Timmusk et al., 1994). Together, our data are important because they suggest that variations in the expression of different MEF2 variants, which occurs both during development and in response to certain kinds of stimuli, can change the transcriptional inducibility of important activity-regulated targets such as $B d n f$, providing a mechanism for the fine-tuning of neuronal transcription-dependent plasticity.

\section{References}

Flavell SW, Cowan CW, Kim TK, Greer PL, Lin Y, Paradis S, Griffith EC, Hu LS, Chen C, Greenberg ME (2006) Activity-dependent regulation of MEF2 transcription factors suppresses excitatory synapse number. Science 311:1008-1012.

Flavell SW, Kim TK, Gray JM, Harmin DA, Hemberg M, Hong EJ, Markenscoff-Papadimitriou E, Bear DM, Greenberg ME (2008) Genome-wide analysis of MEF2 transcriptional program reveals synaptic target genes and neuronal activity-dependent polyadenylation site selection. Neuron 60:1022-1038.

Gossett LA, Kelvin DJ, Sternberg EA, Olson EN (1989) A new myocytespecific enhancer-binding factor that recognizes a conserved element associated with multiple muscle-specific genes. Mol Cell Biol 9:5022-5033.

Hong EJ, McCord AE, Greenberg ME (2008) A biological function for the neuronal activity-dependent component of Bdnf transcription in the development of cortical inhibition. Neuron 60:610-624.

Kornhauser JM, Cowan CW, Shaywitz AJ, Dolmetsch RE, Griffith EC, Hu LS, Haddad C, Xia Z, Greenberg ME (2002) CREB transcriptional activity in neurons is regulated by multiple, calcium-specific phosphorylation events. Neuron 34:221-233.

Loebrich S, Nedivi E (2009) The function of activity-regulated genes in the nervous system. Physiol Rev 89:1079-1103.

Lyons GE, Micales BK, Schwarz J, Martin JF, Olson EN (1995) Expression of mef 2 genes in the mouse central nervous system suggests a role in neuronal maturation. J Neurosci 15:5727-5738.

Lyons MR, West AE (2011) Mechanisms of specificity in neuronal activityregulated gene transcription. Prog Neurobiol 94:259-295.

McDowell KA, Hutchinson AN, Wong-Goodrich SJ, Presby MM, Su D, Rodriguiz RM, Law KC, Williams CL, Wetsel WC, West AE (2010) Reduced cortical BDNF expression and aberrant memory in carf knock-out mice. J Neurosci 30:7453-7465.

Pfenning AR, Kim TK, Spotts JM, Hemberg M, Su D, West AE (2010) Genome-wide identification of calcium-response factor $(\mathrm{CaRF})$ binding sites predicts a role in regulation of neuronal signaling pathways. PLoS One 5:e10870.

Pruunsild P, Sepp M, Orav E, Koppel I, Timmusk T (2011) Identification of cis-elements and transcription factors regulating neuronal activitydependent transcription of human BDNF gene. J Neurosci 31:3295-3308.

Shalizi A, Bonni A (2008) Not just for muscle anymore: activity and calcium regulation of mef2-dependent transcription in neuronal survival and differentiation. In: Transcriptional regulation by neuronal activity (Dudek SM, ed), pp 229-250. New York: Springer.

Shalizi AK, Bonni A (2005) brawn for brains: the role of MEF2 proteins in the developing nervous system. Curr Top Dev Biol 69:239-266.

Shalizi A, Gaudillière B, Yuan Z, Stegmüller J, Shirogane T, Ge Q, Tan Y, Schulman B, Harper JW, Bonni A (2006) A calcium-regulated MEF2 sumoylation switch controls postsynaptic differentiation. Science 311: 1012-1017.

Tao X, Finkbeiner S, Arnold DB, Shaywitz AJ, Greenberg ME (1998) Ca2+ influx regulates BDNF transcription by a CREB family transcription factor-dependent mechanism. Neuron 20:709-726.

Tao X, West AE, Chen WG, Corfas G, Greenberg ME (2002) A calciumresponsive transcription factor, $\mathrm{CaRF}$, that regulates neuronal activitydependent expression of BDNF. Neuron 33:383-395.

Timmusk T, Palm K, Metsis M, Reintam T, Paalme V, Saarma M, Persson H (1993) Multiple promoters direct tissue-specific expression of the rat BDNF gene. Neuron 10:475-489.

Timmusk T, Belluardo N, Persson H, Metsis M (1994) Developmental regulation of brain-derived neurotrophic factor messenger RNAs transcribed from different promoters in the rat brain. Neuroscience 60:287-291.

Zhu B, Gulick T (2004) Phosphorylation and alternative pre-mRNA splicing converge to regulate myocyte enhancer factor $2 \mathrm{C}$ activity. Mol Cell Biol 24:8264-8275. 